\title{
Mortalidad en prematuros tratados con surfactante exógeno
}

\author{
R. MAURICIO BARRÍA P. ${ }^{1}$, PAULINA PINO Z. ${ }^{2}$, CARLOS BECERRA F. ${ }^{3}$
}

1. Enfermero, M.Sc. Epidemiología Clínica. Académico Facultad de Medicina, Universidad Austral de Chile. Becario Proyecto Mecesup AUS 0209, Programa de Doctorado en Salud Pública, Escuela de Salud Pública, Universidad de Chile.

2. Cirujano Dentista, MPH, Dr.PH. Profesora Asociada, División de Epidemiología, Escuela de Salud Pública, Universidad de Chile.

3. Médico Pediatra, Jefe Departamento de Ciclo Vital, Encargado Programa de Salud del Niño, Ministerio de Salud de Chile.

\begin{abstract}
Mortality in premature infants treated with exogenous surfactant

Objective: Characterize mortality and associated factors in Chilean prematures born $\leq 32$ weeks of gestational age (GA) and treated with exogenous surfactant. Method: Cohort of newborns $(n=2868)$ registered between 1998-2005 in the database of the Surfactant National Program. The association of gestational and neonatal variables with mortality was estimated through survival analysis and logistic regression. Results: Global mortality was 35\%, varying by GA from $86.7 \%$ ( $<25$ weeks) to $12.6 \%$ (32 weeks). There was a clear decrease of mortality during the study period, along with a fall in the gestational age and birth weight (BW) of the patients who died (1 $021 \mathrm{~g} \pm 295$ to $854 \mathrm{~g} \pm 258)$ and $\mathrm{GA}(27.7 \pm 2.1$ to 26.5 \pm 23 ) during this period. Pulmonary hemorrhage $(\mathrm{PH})$ was the most important factor associated to mortality, so we decided to stratify the analysis by this condition. In children with $\mathrm{PH}$, the mortality estimated risk lower as the GA increased (OR= 0.73; CI95 0.57-0.93) and every $100 \mathrm{~g}$ of additional BW (OR= 0.74; CI95 0.630.88). Children not affected by PH also had their OR diminished with major GA (OR=0.82; CI95 0.76-0.90) and more BW (OR= 0.84; CI95 0.79 - 0.89). In addition, the OR decreased with better Apgar 5 min score (OR= 0.80 ; CI95 0.75-0.85), use of prenatal corticoids ( $\mathrm{OR}=0.71$; CI95 0.56-0.90) and was higher in boys (OR=1.36; CI95 1.08-1.71). Conclusions: Mortality in premature newborns decreased $15 \%$ during this period. Inmaturity and extreme low birth weight factors constitute a challenge to improve survival and avoid further complications like $\mathrm{PH}$.

(Key words: Infant newborn, infant premature, mortality, neonatal mortality, pulmonary surfactant, risk factors).

Rev Chil Pediatr 2008; 79 (1): 36-44
\end{abstract}

\section{RESUMEN}

Objetivo: Caracterizar la mortalidad y factores asociados en prematuros chilenos $\leq 32$ semanas de edad gestacional (EG) receptores de surfactante exógeno. Pacientes y Métodos: Cohorte de neonatos 1998-2005

Trabajo recibido el 26 de junio de 2007, devuelto para corregir el 30 de julio de 2007, recepción de segunda versión el 24 de agosto de 2007, aceptado para publicación el 10 de noviembre de 2007.

Correspondencia a:

Mauricio Barría P.

E-mail: rbarria@uach.cl 
( $n=2$ 868) de la base de datos del Programa Nacional de Surfactante. Se estimó la mortalidad y su relación con variables maternas, del embarazo y neonatales mediante análisis de sobrevida y regresión logística. Resultados: La mortalidad global fue 35\%, variando por EG entre 86,7\% (< 25 semanas) y 12,6\% (32 semanas). La mortalidad descendió en el período, reduciéndose también el peso de nacimiento (PN) de los fallecidos (1 021g \pm 295 a $854 \mathrm{~g} \pm 258)$ y su EG $(27,7 \pm 2,1$ a 26,5 $\pm 2,3)$. La hemorragia pulmonar (HP) fue el factor más importante asociado a mortalidad, por lo que se estratificó el análisis por esa condición. En niños con HP, cada semana adicional de EG disminuye el riesgo de morir (OR: 0,73; $\mathrm{IC}_{95}$ 0,57-0,93), así como por cada 100 g de peso adicional (OR: 0,74; $\mathrm{IC}_{95}$ 0,63-0,88). Sin HP, el riesgo disminuye con mayor EG (OR: 0,82; $\mathrm{IC}_{95}$ 0,76-0,90), mayor PN (OR: 0,84; $\mathrm{IC}_{95}$ 0,79-0,89), mejor puntuación Apgar 5 minutos (OR: 0,80; IC 95 0,750,85) y uso de corticoide prenatal (OR: 0,71; $\mathrm{IC}_{95}$ 0,56-0,90), siendo significativamente mayor en varones (OR: 1,36; $\mathrm{IC}_{95}$ 1,08-1,71). Conclusiones: En el período, la mortalidad en prematuros disminuyó en $15 \%$. La inmadurez y extremo bajo peso de niños actualmente viables, plantean importantes desafíos para mejorar su sobrevida y evitar complicaciones, entre ellas, la HP.

(Palabras clave: Factor de riesgo, Mortalidad, Mortalidad neonatal, Prematuro, Recién nacido, Surfactante pulmonar).

Rev Chil Pediatr 2008; 79 (1): 36-44

\section{Introducción}

En los últimos años la mortalidad infantil (MI) en Chile ha disminuido de 16 por mil nacidos vivos en 1990 a 8,4 por mil en 2004, fundamentalmente por reducción de la MI tardía ${ }^{1}$. Aunque la mortalidad neonatal (MNN) también se redujo, su descenso ha sido menor. Entre 1998 y 2004 ocurrieron en el país 21549 nacimientos entre 22 y 32 semanas de gestación evidenciándose un aumento de recién nacidos (RN) menores de 25 semanas $^{2}$. A la par de este incremento, la sobrevida de prematuros extremos y de niños de muy bajo peso ha mejorado significativamente, gracias a los avances en la medicina perinatal, como a la reorganización de los cuidados y nuevas terapias neonatales ${ }^{3,4}$. Concomitantemente se ha producido un desplazamiento de la edad de fallecimiento de una parte de estos neonatos ${ }^{5}$.

Los trastornos neonatales encabezan las causas de muerte en menores de un año incluyendo malformaciones cardiacas y del sistema nervioso, trastornos de la gestación y del crecimiento fetal, y dificultad respiratoria ${ }^{6}$.

El síndrome de distrés respiratorio (SDR) ha sido descrito como la causa más común de falla respiratoria y de indicación de ventilación mecánica en RN y permanece como una importante causa de morbimortalidad neonatal ${ }^{7}$. En Chile afecta hasta $60 \%$ de los menores de $1000 \mathrm{~g}^{5}$ alcanzando 4\% de la $\mathrm{MI}^{6}$.
El uso de corticoides prenatales (CPN) para inducir maduración pulmonar y la introducción del surfactante exógeno, han sido trascendentales en los logros alcanzados en estos niños. Desde su introducción hace más de 20 años, muchos han sido los niños tratados con surfactante, convirtiéndose en tratamiento de rutina en la práctica neonatal. Sus efectos y mecanismos de acción han sido ampliamente descritos ${ }^{8-10}$, manteniéndose como una intervención efectiva y segura como profilaxis y rescate de niños con SDR, reduciendo la morbimortalidad ${ }^{11}$. Aunque es difícil aislar el efecto del CPN, internacionalmente se reconoce que la mejor sobrevida de neonatos con SDR es atribuible al tratamiento con surfactante, aún considerando la incrementada incidencia de niños de riesgo $0^{3,8,12}$.

En 1998 el Ministerio de Salud de Chile implementó el Programa Nacional de Surfactante que incorpora los datos aportados por los centros del sistema público que cuentan con cuidado intensivo neonatal. Los resultados del programa no han sido bien dimensionados, pero se asume que parte de la reducción de la MNN puede ser considerada una señal de la eficacia de esta política nacional ${ }^{13}$. En este contexto, la necesidad de información rigurosa y oportuna es esencial para el análisis de intervenciones de tal envergadura.

Basado en la información disponible, el objetivo del estudio fue estimar la mortalidad en prematuros de hasta 32 semanas que recibie- 
ron surfactante exógeno y estimar el efecto (odds ratio, OR) de factores de riesgo específicos en el desenlace de muerte.

\section{Pacientes y Métodos}

Se empleó la base de datos del Programa Nacional de Surfactante la cual es construida a partir de la información entregada por los centros pertenecientes a hospitales públicos del país. Se utilizó los datos de neonatos prematuros nacidos entre abril de 1998 y octubre de 2005. Aún cuando esta base de datos incorpora un gran número de observaciones, es necesario aclarar que el reporte de la información requerida por el programa desde los diferentes hospitales fue realizado de manera diferencial en los distintos años y desde los diferentes centros. Por lo tanto, los análisis comparativos y la representatividad por año deben ser considerados en este contexto.

De 3796 RN de los que se dispuso información, 2887 tenían edad gestacional (EG) $\leq 32$ semanas. Se excluyó observaciones con datos inconsistentes o sin especificación de la condición de egreso, conformándose finalmente una cohorte de 2868 niños. Todos estos neonatos recibieron como terapia surfactante alguno de los 2 productos dispuestos por el programa ministerial durante el periodo de estudio, es decir, Exosurf ${ }^{\circledR}$ y Survanta ${ }^{\circledR}$.

Se definió como resultado la condición de egreso (vivo/fallecido) analizándose su relación con edad materna, rotura prematura de membranas (RPM), síndrome hipertensivo del embarazo (SHIE), peso de nacimiento (PN), EG, sexo, uso de CPN, tipo de parto, Apgar al minuto (Apgar 1') y cinco minutos (Apgar 5'), dosis, tipo y momento de administración de surfactante y hemorragia pulmonar (HP). Variables categóricas como sexo, tipo de parto, antecedentes de RPM y SHIE, uso de CPN, tipo de surfactante y HP se describen como proporción e intervalo de confianza 95\% ( $\left.\mathrm{IC}_{95}\right)$, en tanto que edad materna, PN, EG, Apgar, tiempo a la administración de primera dosis de surfactante -variables continuas u ordinalesse presentan como promedio y desviación estándar ( $\pm \mathrm{DE})$, o mediana y rango intercuartil [p25-p75] cuando la distribución no fue normal. La valoración de asociación con el desenlace (mortalidad) se estimó mediante prueba de $\chi^{2}$ o riesgo relativo (RR) para variables categóricas y prueba de Student (t-test) o de Mann-Whitney para las continuas. Fue utilizada la variable “días de estada" como definición de tiempo de seguimiento para cálculo de la sobrevida, estimándose curvas Kaplan-Meier por categorías de EG y comparando con prueba Log-rank $(\alpha 0,05)$. Como estimación univariada del riesgo de muerte se calculó razones de disparidad (odds ratio, OR) y su $\mathrm{IC}_{95}$ a partir de regresión logística simple (OR crudos). Mediante regresión logística múltiple (RLM) se controló el efecto conjunto de las variables de interés (OR ajustados). El uso de CPN fue informado en 2835 (98,8\%) niños, total sobre el cual se efectuó el análisis multivariado. Se utilizó el programa Stata versión 8.1 (Stata Corporation, College Station, Texas, USA 2003).

\section{Resultados}

\section{Condiciones generales}

El PN y EG (media \pm DE) fueron $1174 \mathrm{~g}$ $( \pm 385)$ y 28,6 semanas $( \pm 2,3)$ respectivamente. Extremo bajo peso (menos de $1000 \mathrm{~g}$ ) se presentó en 38,7\%, mientras 49,4\% no superaron las 28 semanas. Nacimiento por cesárea se registró en $61,3 \%$, proporción que se mantuvo estable entre 1998 y 2005 alcanzando su máximo en 2001 (65,5\%). La puntuación media del Apgar 1' y 5' fue 5,2 ( $\pm 2,7)$ y 7,2 ( $\pm 1,9)$ respectivamente.

La edad materna promedio fue 26,4 años $( \pm 7,5)$ y se consignó $27 \%$ de RPM y $22,4 \%$ de SHIE. 59,1\% de las madres recibió CPN, administrándose 2 dosis al $64,3 \%$ de ellas. Se incrementó en $72 \%$ el uso de CPN entre 1998 y 2005 (de 43,3\% a 74,6\%).

La incidencia de HP fue 10,3\% (294 casos). La EG media fue significativamente menor en quienes desarrollaron dicha condición, 27,8 $( \pm 2,1)$ vs 28,8 $( \pm 2,3)$ semanas $(p=0,000)$, siendo su incidencia máxima en prematuros de 24 semanas (18,3\%) (figura 1). De los datos analizados, podría especularse que, aunque esta patología disminuyó discretamente en algunos 


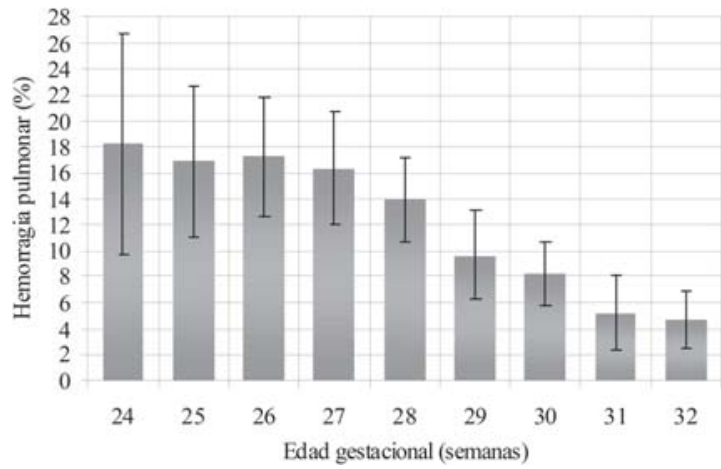

Figura 1. Incidencia de hemorragia pulmonar (porcentaje e $\mathrm{IC}_{95}$ ) según edad gestacional.

años, permaneció más bien estable, alcanzando $11,2 \%$ en 1998 y $11,3 \%$ en 2005 , comportamiento que se mantuvo por grupos de EG.

\section{Mortalidad}

La mortalidad global alcanzó 35\% variando entre 86,7\% (91/105) en menores de 25 semanas y $12,6 \%$ en niños de 32 semanas (tabla 1 ). Los fallecidos presentaron significativamente menor PN y EG, uso de CPN y parto por cesárea; fueron mayoritariamente de sexo masculino y presentaron significativa mayor proporción de Apgar bajo al minuto y cinco minutos, así como, mayor incidencia de HP. No hubo diferencias de la mortalidad según dosis de surfactante recibidas (tabla 2).

El análisis de tendencia anual evidenció una
Tabla 1. Mortalidad por edad gestacional

\begin{tabular}{|c|c|c|c|c|}
\hline \multirow[b]{2}{*}{$\begin{array}{l}\text { Edad } \\
\text { gestacional }\end{array}$} & \multirow[b]{2}{*}{ Total } & \multicolumn{3}{|c|}{ Fallecidos } \\
\hline & & $\mathbf{n}$ & $\%$ & $\begin{array}{c}\% \\
\text { acumulado }\end{array}$ \\
\hline 22 & 3 & 3 & 100 & 0,3 \\
\hline 23 & 10 & 8 & 80,0 & 1,1 \\
\hline 24 & 92 & 80 & 87,0 & 9,1 \\
\hline 25 & 204 & 148 & 72,5 & 23,8 \\
\hline 26 & 297 & 191 & 64,3 & 42,8 \\
\hline 27 & 304 & 145 & 47,7 & 57,2 \\
\hline 28 & 506 & 169 & 33,4 & 74,0 \\
\hline 29 & 296 & 76 & 25,7 & 81,6 \\
\hline 30 & 512 & 101 & 19,7 & 91,6 \\
\hline 31 & 263 & 36 & 13,7 & 95,2 \\
\hline 32 & 381 & 48 & 12,6 & 100,0 \\
\hline Total & 2868 & 1005 & 35,0 & \\
\hline
\end{tabular}

reducción de la mortalidad, situándose bajo 30\% a partir de 2002. Aunque se registró un leve incremento en 2005, no se retomaron los valores iniciales. Se observó una reducción relativa de $15,3 \%$ en la mortalidad durante el período. La EG media de los fallecidos cayó de 27,7 \pm 2,1 en 1998 a 26,5 $\pm 2,3$ en 2005. Consecuentemente se redujo el peso medio de los fallecidos llegando hasta $854 \mathrm{~g} \pm 258$ en 2005 (tabla 3). Las modificaciones en los criterios de administración de surfactante en términos de PN y EG en el transcurso del tiempo, estarían determinando la reducción de la media de estas variables en los fallecidos. En términos de peso, la proporción de menores de $750 \mathrm{~g}$ se ha incrementado desde $10,6 \%$ en el periodo 19982000 hasta $12,3 \%$ entre 2001-2005, destacando

Tabla 2. Características de los neonatos según condición de egreso

\begin{tabular}{|c|c|c|c|}
\hline Variable & $\begin{array}{c}\text { Vivo } \\
\mathrm{n}=1863\end{array}$ & $\begin{array}{l}\text { Fallecido } \\
n=1005\end{array}$ & $\mathbf{p}$ \\
\hline PN (g) Media \pm DE & $1289 \pm 365$ & $960 \pm 325$ & $0,0000^{*}$ \\
\hline EG (semanas) Media $\pm \mathrm{DE}$ & $29,3 \pm 1,9$ & $27,2 \pm 2,1$ & $0,0000^{*}$ \\
\hline Apgar 1 minuto, puntuación $\leq 3(\%)$ & 22,7 & 44,9 & $0,000^{\ddagger}$ \\
\hline Apgar 5 minutos, puntuación $\leq 6(\%)$ & 18,5 & 41,8 & $0,000^{\ddagger}$ \\
\hline Uso de CPN (\%) & 63,9 & 51,4 & $0,000^{\ddagger}$ \\
\hline Sexo masculino (\%) & 55,8 & 61,3 & $0,005^{\ddagger}$ \\
\hline Cesárea (\%) & 64,2 & 57 & $0,000^{\ddagger}$ \\
\hline \multicolumn{4}{|l|}{ Uso de surfactante } \\
\hline Dosis única (\%) & 29,8 & 30,1 & $N S^{\ddagger}$ \\
\hline Tiempo a la $1^{a}$ dosis $(\mathrm{h})$ Mediana $[\mathrm{RIC}]$ & $3[2-6]$ & $2[1-5]$ & $0,0000^{\dagger}$ \\
\hline HP (\%) & 4,0 & 21,8 & $0,000^{\ddagger}$ \\
\hline
\end{tabular}

PN: peso de nacimiento. EG: edad gestacional. CPN: corticoide prenatal. HP: hemorragia pulmonar. DE: desviación estándar; RIC: rango intercuartil. NS: no significativo. "Prueba t de Student; ${ }^{\dagger}$ Prueba de Mann-Whitney; APrueba $^{2}$ 
que para 2004 y 2005 llegó a 15,8 y 14,5\%, respectivamente. Al analizar la proporción de niños nacidos con menos de 26 semanas de gestación en los distintos años se observó un patrón similar, variando desde 7,9 y 10,2\% en los años 1998 y 1999 hasta 14,5 y 15,3\% en 2004 y 2005. No obstante, cabe resaltar que aun cuando el grupo de neonatos tratados con surfactante en los últimos años incorpora niños de menor PN y EG, se ha mantenido la tendencia de reducción de mortalidad.

El RR de muerte en niños de extremo bajo peso fue 3,0 ( $\left.\mathrm{IC}_{95} 2,7-3,4\right)$ y hubo una considerable diferencia de sobrevida en niños con EG $\leq 28$ semanas (figura 2). El riesgo de muerte fue 3 veces mayor en estos niños $(\mathrm{RR}=2,9$; $\mathrm{IC}_{95}$ 2,6-3,3). En particular, en niños $<27$ semanas la probabilidad de sobrevida en los primeros días cayó drásticamente por cada sema- na menos de gestación (figura 3). Independientemente de la EG, la muerte ocurrió en la mayoría de los casos dentro de los primeros días: $52 \%$ en el primer día y 54\% hasta el tercero.

\section{Uso de surfactante y mortalidad}

Durante el periodo estudiado el programa ministerial empleó un surfactante sintético (Exosurf $\left.{ }^{\circledR}\right)$ y/o uno natural (Survanta $\left.{ }^{\circledR}\right)$, este último usado de manera exclusiva desde 2004. Los neonatos que recibieron Survanta ${ }^{\circledR}$ evidenciaron menor mortalidad en los grupos de EG > 25 semanas (figura 4), pero las diferencias fueron significativas sólo en los grupos de $25-26$ ( $p=0,02)$ y de 29-30 semanas ( $p=0,007)$. Asimismo, $10 \%$ de los niños que recibieron Survanta ${ }^{\circledR}$ y $14 \%$ de los tratados con Exosurf® presentaron HP $(p=0,000)$.

Tabla 3. Mortalidad neonatal anual según peso al nacer y edad gestacional de los fallecidos

\begin{tabular}{|c|c|c|c|c|c|}
\hline Año & $\begin{array}{c}\text { Total } \\
n=2868\end{array}$ & $\begin{array}{l}\text { Fallecidos } \\
n=1005\end{array}$ & $\begin{array}{c}\text { Mortalidad } \\
(\%)\end{array}$ & $\begin{array}{c}\text { EG (semanas) } \\
\text { Media } \pm \mathrm{DE}\end{array}$ & $\begin{array}{c}\text { PN (g) } \\
\text { Media } \pm \text { DE }\end{array}$ \\
\hline 1998 & 430 & 163 & 37,9 & $27,7 \pm 2,1$ & $1021 \pm 295$ \\
\hline 1999 & 578 & 232 & 40,1 & $27,4 \pm 2,2$ & $975 \pm 305$ \\
\hline 2000 & 679 & 244 & 35,9 & $27,2 \pm 2,2$ & $965 \pm 355$ \\
\hline 2001 & 445 & 154 & 34,6 & $27,1 \pm 2,1$ & $937 \pm 353$ \\
\hline 2002 & 305 & 87 & 28,5 & $26,8 \pm 2,0$ & $900 \pm 261$ \\
\hline 2003 & 69 & 17 & 24,6 & $26,9 \pm 2,6$ & $933 \pm 374$ \\
\hline 2004 & 228 & 63 & 27,6 & $27,2 \pm 2,6$ & $940 \pm 362$ \\
\hline 2005 & 131 & 42 & 32,1 & $26,5 \pm 2,3$ & $854 \pm 258$ \\
\hline $\mathrm{NC}$ & 3 & 3 & - & - & - \\
\hline
\end{tabular}

EG: edad gestacional. PN: peso de nacimiento. NC: no consignado

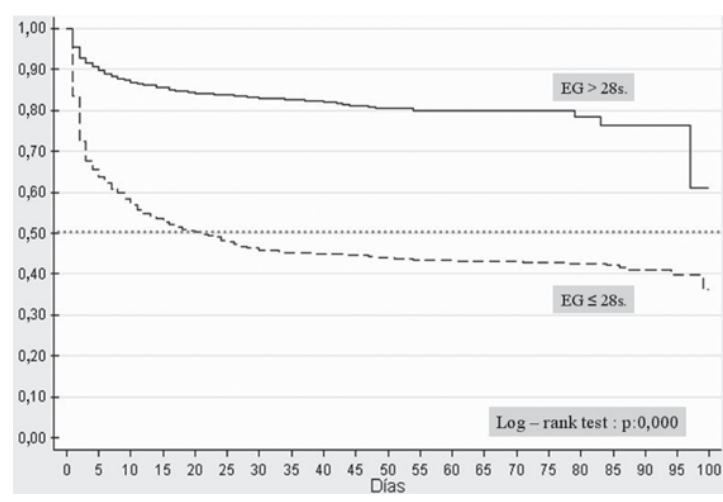

Figura 2. Estimación de Sobrevida (Kaplan-Meier) en neonatos de más y menos de 28 semanas de gestación durante primeros 100 días.

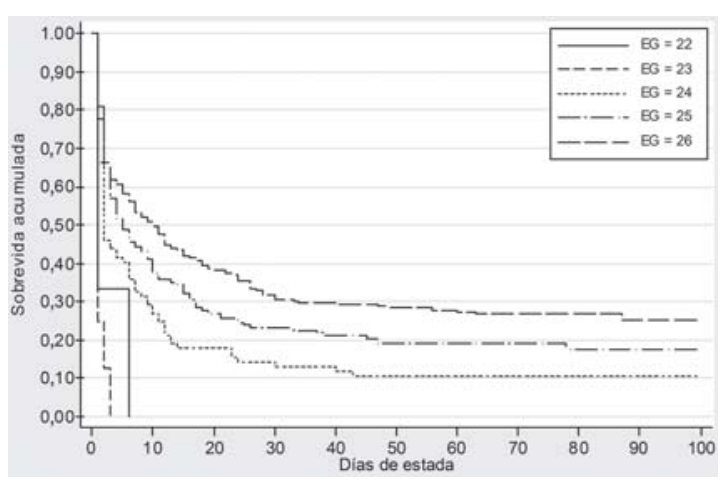

Figura 3. Estimación de Sobrevida (Kaplan-Meier) en prematuros bajo 27 semanas de gestación durante primeros 100 días. 


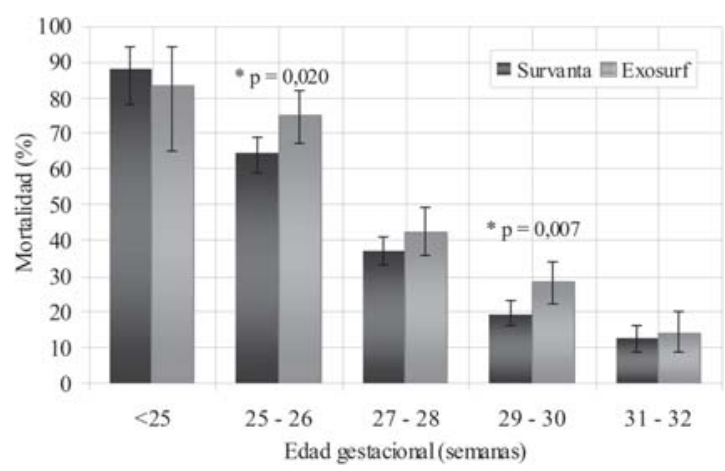

Figura 4. Mortalidad (proporción e intervalo de confianza 95\%) por edad gestacional según tipo de surfactante utilizado.

Casi un tercio (29,4\%) de los niños recibió dosis única, criterio que ha ganado preponderancia en el período, pasando de $24 \%$ en 1998 a $51,1 \%$ en 2005 . No hubo diferencias en la mortalidad según el número de dosis administradas.

El tiempo mediano de administración de la primera dosis fue $3 \mathrm{~h}$, disminuyendo de 5 a $2 \mathrm{~h}$ en los últimos años, reduciéndose también la variabilidad. En niños con EG $\leq 28$ semanas este tiempo fue significativamente menor, $2 \mathrm{~h}$ [1-4] vs 4 h [2-7] $(\mathrm{p}=0,000)$.

\section{Factores de riesgo de mortalidad}

La mortalidad se asocia con HP, uso de CPN, puntuación Apgar, EG, PN, sexo, tipo y tiempo de administración de surfactante (tabla 4-A). Sin embargo, consideramos que, más que un factor de riesgo, la HP seguramente constituye una variable intermedia entre otros factores y la mortalidad, lo que llevó a estratificar por categorías de HP (presente o ausente). Controlando el efecto de variables maternas, del embarazo y perinatales (modelo ajustado), se obtuvo que sólo la EG y el PN se asocian significativamente con la mortalidad en RN que desarrollaron HP, reduciéndose en alrededor de $25 \%$ el OR de muerte por cada semana de gestación adicional y por cada $100 \mathrm{~g}$ de peso. En RN sin HP, además del PN y EG, el uso de CPN y mayor puntuación Apgar 5'reducen significativamente el riesgo de morir, en tanto aumenta $36 \%$ en varones (tabla 4-B). El tipo y número de dosis de surfactante muestran un efecto reductor en el límite de la significancia estadística.

Tabla 4. Factores asociados a mortalidad en prematuros de EG $\leq 32$ semanas receptores de surfactante pulmonar. Análisis univariado y multivariado estratificado por presencia [HP (+)] o ausencia [HP (-)] de hemorragia pulmonar

\begin{tabular}{|c|c|c|c|c|c|c|}
\hline \multirow[t]{3}{*}{ Variable } & \multirow{2}{*}{\multicolumn{2}{|c|}{$\frac{\text { A }}{\text { Análisis univariado }}$}} & \multicolumn{4}{|c|}{$\begin{array}{c}\text { B } \\
\text { Análisis multivariado* }\end{array}$} \\
\hline & & & \multicolumn{2}{|c|}{ Modelo HP (-) } & \multirow{2}{*}{$\begin{array}{l}\text { Model } \\
\text { ORa }^{\dagger}\end{array}$} & \multirow{2}{*}{$\begin{array}{l}\text { HP (+) } \\
\text { IC }_{95 \%}\end{array}$} \\
\hline & OR & $I_{95 \%}$ & ORa $^{\dagger}$ & IC $_{95 \%}$ & & \\
\hline Edad materna (por año) & 1,0 & $0,99-1,00$ & 0,99 & $0,98-1,01$ & 1,03 & $0,98-1,08$ \\
\hline Cesárea & 0,74 & $0,63-0,86$ & 1,0 & $0,78-1,29$ & 0,93 & $0,38-2,29$ \\
\hline CPN & 0,58 & $0,50-0,68$ & 0,71 & $0,56-0,90^{\ddagger}$ & 0,77 & $0,34-1,75$ \\
\hline RPM & 1,11 & $0,93-1,32$ & 0,98 & $0,75-1,27$ & 0,85 & $0,34-2,10$ \\
\hline SHIE & 0,80 & $0,66-0,96$ & 0,74 & $0,53-1,03$ & 1,05 & $0,42-2,63$ \\
\hline Apgar 5 minutos (por c/punto) & 0,72 & $0,69-0,75$ & 0,80 & $0,75-0,85^{\ddagger}$ & 0,88 & $0,72-1,07$ \\
\hline EG (por c/semana) & 0,63 & $0,61-0,66$ & 0,82 & $0,76-0,90^{\ddagger}$ & 0,73 & $0,57-0,93^{\ddagger}$ \\
\hline PN (por c/100 g) & 0,74 & $0,72-0,76$ & 0,84 & $0,79-0,89^{\ddagger}$ & 0,74 & $0,63-0,88^{\ddagger}$ \\
\hline $\begin{array}{l}\text { Sexo masculino } \\
\text { Uso de Surfactante }\end{array}$ & 1,25 & $1,07-1,47$ & 1,36 & $1,08-1,71^{\ddagger}$ & 1,12 & $0,54-2,31$ \\
\hline Survanta ${ }^{\circledR}$ & 0,77 & $0,65-0,91$ & 0,78 & $0,61-1,00$ & 1,71 & $0,84-3,48$ \\
\hline Tiempo $1^{\circ}$ dosis (por $\left.\mathrm{c} / \mathrm{h}\right)$ & 0,97 & $0,95-0,98$ & 0,99 & $0,98-1,01$ & 0,97 & $0,91-1,03$ \\
\hline Dosis (2 o más) & 0,98 & $0,83-1,16$ & 0,77 & $0,60-1,00$ & 1,05 & $0,44-2,5$ \\
\hline $\mathrm{HP}$ & 7,80 & $5,9-10,20$ & - & - & - & - \\
\hline
\end{tabular}

CPN: corticoide prenatal. RPM: rotura prematura de membranas. SHIE: síndrome hipertensivo inducido por el embarazo. EG: edad gestacional. PN: peso de nacimiento. HP: hemorragia pulmonar. ${ }^{*}$ Regresión Logística Múltiple. ${ }^{+}$Odds ratio ajustado (ORa). ${ }^{\ddagger} p<0,05$. 


\section{Discusión}

Durante el período estudiado se redujo la mortalidad y disminuyó el promedio del PN y EG de los neonatos fallecidos, sugiriendo un efecto de las mejorías en la medicina perinatal. Entre éstas, se ha constatado la optimización de la terapia surfactante y el aumento de la cobertura del CPN.

Concordante con la literatura, se constató que independientemente de otras causas, la HP es un trastorno que una vez instalado incrementa drásticamente el riesgo de muerte. Un estudio norteamericano registró 68\% de HP en neonatos fallecidos durante la primera semana ${ }^{14}$ y Narayan et $\mathrm{al}^{15}$ reportaron $30 \%$ de HP en fallecidos versus $3 \%$ en sobrevivientes, cifras similares a las de nuestro estudio. La estabilidad de la incidencia de HP mostrada en el periodo difiere de los resultados de la Neonatal Research Network of National Institute of Child health \& Human Development (NICHD) que registran para EEUU un aumento a partir de $1998^{16}$. Al igual que en nuestro estudio, se ha reportado que los fallecidos con HP tienen significativamente menos PN y menor $\mathrm{EG}^{17}$.

Así como otros estudios nacionales, verificamos que la mortalidad ocurre precozmente, fundamentalmente en el primer día 5 . El incremento de sobrevida por cada semana adicional, especialmente bajo 27 semanas, está vinculada a la corrección de la incompetencia pulmonar ${ }^{18}$ y es justamente este grupo el que más se beneficiaría con el surfactante ${ }^{10}$. La EG demuestra su capacidad de predictor de sobrevida, incluso superior al $\mathrm{PN}^{18,19}$. Es un factor sustancial para decidir la oportunidad del parto en embarazos de riesgo ${ }^{5}$ pero requiere ser conocido previamente y válidamente estimado.

La reducción del riesgo de muerte con la mejor puntuación Apgar 5' concuerda con resultados previamente reportados en neonatos de muy bajo peso con membrana hialina $(\mathrm{OR}=$ $0,80 ; \mathrm{p}=0,0166)^{20}$. Casey et $\mathrm{al}^{21}$ encontraron alto riesgo de muerte asociado a su baja puntuación entre las 26 y 36 semanas para valores entre 0 y 3 puntos ( $R R=59$; $\left.I_{95} 40-87\right)$, y entre 4 y $6\left(R R=13 ; I_{95}\right.$ 9-20). También fue un factor significativo para la sobrevida de neonatos de cuidados intensivos en Brasil en- contrándose un Hazard Ratio de 1,8 ( $\mathrm{IC}_{95}$ 1,02,45 ) en puntuación de 4-6 y de 2,25 (IC I $_{95} 1,29$ $5,34)$ entre $0-3^{22}$. No obstante, dada la influencia de otros factores en el Apgar, éste no puede ser considerado aisladamente para decidir el manejo clínico o como evidencia de asfixia o predictor de resultados neurológicos ${ }^{23,24}$.

La asociación de mortalidad y sexo masculino encontrada en niños que no desarrollaron HP, es una condición que ha sido documentada. Glinianaia et $\mathrm{al}^{25}$ reportaron reducción de la mortalidad en niños de ambos sexos aunque persistiendo un mayor riesgo en varones (RR: 1,26; $\left.\mathrm{IC}_{95} 1,18-1,36\right)$. Similarmente, se registró un OR 1,7 para RN masculinos de extremo bajo peso ${ }^{26,27}$ y Morse et $\mathrm{al}^{28}$ encontraron un incremento de la sobrevida en niñas independiente de la raza $\left(\mathrm{OR}=1,7 ; \mathrm{IC}_{95} 1,5-2,0\right)$. Aunque el mecanismo biológico de estas diferencias por sexo no es claro, la desventaja en varones se extiende a aspectos como un mayor requerimiento de soporte ventilatorio y de reanimación, y mayor probabilidad de trastornos respiratorios y circulatorios ${ }^{29,30}$. Como explicación se ha sugerido que factores hormonales determinarían una maduración pulmonar más acelerada en mujeres que las harían menos vulnerables frente a la asfixia y la membrana hialina ${ }^{31}$. Esto concuerda con la caída más marcada de la mortalidad por SDR en prematuros varones encontrada por Bhaumik et $\mathrm{al}^{32}$ la que podría reflejar un mayor beneficio de terapias como el uso de CPN y surfactante en este grupo.

Los mejores resultados alcanzados en las últimas décadas están marcados por los efectos de intervenciones como uso de CPN y terapia surfactante ${ }^{3,33}$. Crowley ${ }^{34}$ describió que el uso de CPN es un importante factor de reducción del riesgo -aproximadamente 50\%de morbilidad por SDR y mortalidad. Por su parte, el uso de surfactante ha sido ampliamente evaluado y tanto estudios experimentales como observacionales han comprobado su eficacia en la prevención y tratamiento del SDR ${ }^{10}$.

Hemos verificado que, en el período, la administración del surfactante se ha hecho más precoz en neonatos de menor EG. Es posible que esta conducta se deba a una más pronta aplicación del protocolo clínico establecido, el 
cual está basado en la evidencia que demuestra su efectividad en la reducción de la severidad, las complicaciones respiratorias y la mortali$\operatorname{dad}^{35}$.

De acuerdo con estos resultados parece acertada la política de uso exclusivo de surfactante natural a partir de 2004, dada la mejor sobrevida observada en niños que recibieron este producto, lo cual ya ha sido previamente reportado ${ }^{10,36-38}$.

Reiteramos que en la valoración de estos resultados debe considerarse el posible sesgo de información y de selección dado el reporte diferencial a lo largo de los años y desde los centros de origen. Debido a esta limitación se desestimó la realización de un análisis por servicios o centros específicos, evaluando factores como el acceso, efectos regionales y otras variables desde una perspectiva ecológica. Reconociendo las limitaciones, consideramos que este estudio devela las modificaciones en el perfil de los neonatos atendidos en el conjunto de las unidades de cuidados intensivos neonatales y los cambios en sus expectativas de sobrevida. Ha permitido también estimar la magnitud del efecto de factores específicos sobre la mortalidad. Estudios adicionales deben considerar las medidas preventivas y terapéuticas de trastornos de alta letalidad, como la HP, a fin de reducir la mortalidad de los prematuros.

Cabe una reflexión final respecto de la importancia de la generación, acceso oportuno y calidad de bases de datos para la evaluación de los programas ministeriales de salud. En este caso, se valora la existencia de este registro nacional pero su máxima trascendencia se lograría con su vinculación -mediante un código identificador- con otros registros nacionales, particularmente los de nacimientos y mortalidad.

\section{Agradecimientos}

Agradecemos al Sr. Héctor Álvarez por la importante colaboración en el estudio. Además valoramos y reconocemos a cada uno de los Jefes de los respectivos centros a lo largo del país por el aporte sustancial en la entrega de información para la creación de la base de datos.

\section{Referencias}

1.- Departamento de Estadísticas e Información de Salud: Mortalidad infantil y sus componentes, Chile 1990-2004. Ministerio de Salud 2006; Disponible en: URL: http://deis.minsal.cl/deis/ev/mortalidad_infantil/ series/consulta04.asp Consultado en Agosto 2006.

2.- Departamento de Estadísticas e Información de Salud: Bases de datos de defunciones y nacimientos. Ministerio de Salud 2006; Disponible en: URL: http:// deis.minsal.cl/ Consultado en Agosto 2006.

3.- Stoelhorst GM, Rijken M, Martens SE, Brand R, Den Ouden $A L$, Wit $J M$, et al: Changes in neonatology: comparison of two cohorts of very preterm infants (gestational age < 32 weeks): the Project On Preterm and Small for Gestational Age Infants 1983 and the Leiden Follow-Up Project on Prematurity 1996-1997. Pediatrics 2005; 115: 396-405.

4.- Cooke RW: Preterm mortality and morbidity over 25 years. Arch Dis Child Fetal Neonatal Ed 2006; 91: F293-4.

5.- Morgues M, Henríquez MT, Tohá D, Vernal P, Pittaluga E, Vega S, et al: Sobrevida del niño menor de 1500g.en Chile. Rev Chil Obstet Ginecol 2002; 67: 100-5.

6.- Departamento de Estadísticas e Información de Salud: Mortalidad de menores de un año, según las principales causas específicas de defunción 2004, Chile. Ministerio de Salud 2006; Disponible en: URL: http:// deis.minsal.cl/deis/ev/mortalidad_infantil/causas/ mort_inf_total.asp Consultado en Agosto 2006.

7.- Ainsworth SB: Pathophysiology of neonatal respiratory distress syndrome: implications for early treatment strategies. Treat Respir Med 2005; 4: 42337.

8.- Curley AE, Halliday HL: The present status of exogenous surfactant for the newborn. Early Hum Dev 2001; 61: 67-83.

9.- Obladen M: History of surfactant up to 1980. Biol Neonate 2005; 87: 308-16.

10.- Ghodrat M: Lung surfactants. Am J Health Syst Pharm 2006; 63: 1504-21.

11.- Halliday $H L$ : Recent clinical trials of surfactant treatment for neonates. Biol Neonate 2006; 89: 3239.

12.- Choi YY, Park JY, Cho CY, Ma JS, Hwang TJ: Changes of neonatal mortality rate between 'pre' and 'post' surfactant period. J Korean Med Sci 1999; 14: 45-51.

13.- González R, Merialdi M, Lincetto O, Lauer J, Becerra $C$, Castro $R$, et al: Reduction in neonatal mortality in Chile between 1990 and 2000. Pediatrics 2006; 117: e949-54.

14.- Kostelanetz AS, Dhanireddy R: Survival of the verylow-birth-weight infants after cardiopulmonary resuscitation in neonatal intensive care unit. J Perinatol 2004; 24: 279-83.

15.- Narayan S, Aggarwal R, Upadhyay A, Deorari AK, Singh $M$, Paul VK: Survival and morbidity in extremely 
low birth weight (ELBW) infants. Indian Pediatr 2003; 40: $130-5$.

16.- St John EB, Carlo WA: Respiratory distress syndrome in VLBW infants: changes in management and outcomes observed by the NICHD Neonatal Research Network. Semin Perinatol 2003; 27: 288-92.

17.- Tomaszewska M, Stork E, Minich Nm, Friedman $H$, Berlin S, Hack M: Pulmonary hemorrhage: clinical course and outcomes among very low-birth-weight infants. Arch Pediatr Adolesc Med 1999; 153: 71521.

18.- Hubner ME, Ramírez R: Sobrevida, viabilidad y pronóstico del prematuro. Rev Méd Chile 2002; 130: 931-8.

19.- Hintz SR, Poole WK, Wright LL, Fanaroff AA, Kendrick $D E$, Laptook $A R$, et al: Changes in mortality and morbidities among infants born at less than 25 weeks during the post-surfactant era. Arch Dis Child Fetal Neonatal Ed 2005; 90: F128-33.

20.- García-Arias MB, Zuluaga AP, Arrabal-Terán MC, Arizcun PJ: Factores de riesgo en la mortalidad de los recién nacidos de muy bajo peso al nacer con membrana hialina. An Pediatr (Barc) 2005; 63: 109-15.

21.- Casey BM, Mcintire DD, Leveno KJ: The continuing value of the Apgar score for the assessment of newborn infants. N Engl J Med 2001; 344: 467-71.

22.- Weirich CF, Andrade AL, Turchi MD, Silva SA, MoraisNeto $O L$, Minamisava $R$, et al: Neonatal mortality in intensive care units of Central Brazil. Rev Saude Publica 2005; 39: 775-81.

23.- Chong DS, Karlberg J: Refining the Apgar score cutoff point for newborns at risk. Acta Paediatr 2004; 93: 53-9.

24.- American Academy of Pediatrics, American College of Obstetricians and Gynecologists:The Apgar score. Pediatrics 2006; 117: 1444-7.

25.- Glinianaia SV, Rankin J, Bell R, Pearce MS, Parker L: Temporal changes in the distribution of population risk factors attenuate the reduction in perinatal mortality. J Clin Epidemiol 2005; 58: 1299-307.

26.- Shankaran S, Fanaroff AA, Wright LL, Stevenson DK, Donovan EF, Ehrenkranz RA, et al: Risk factors for early death among extremely low-birth-weight infants. Am J Obstet Gynecol 2002; 186: 796-802.

27.- Bacak SJ, Baptiste-Roberts K, Amon E, Ireland B,
Leet T: Risk factors for neonatal mortality among extremely-low-birth-weight infants. Am J Obstet Gynecol 2005; 192: 862-7.

28.- Morse SB, Wu SS, Ma C, Ariet M, Resnick M, Roth J: Racial and gender differences in the viability of extremely low birth weight infants: a population-based study. Pediatrics 2006; 117: e106-12.

29.- Stevenson DK, Verter J, Fanaroff $A A, O h W$, Ehrenkranz RA, Shankaran $S$, et al: Sex differences in outcomes of very low birthweight infants: the newborn male disadvantage. Arch Dis Child Fetal Neonatal Ed 2000; 83: F182-5.

30.- Elsmen E, Hansen PI, Hellstrom-Westas L: Preterm male infants need more initial respiratory and circulatory support than female infants. Acta Paediatr 2004; 93: 529-33.

31.- Nielsen HC: Testosterone regulation of sex differences in fetal lung development. Proc Soc Exp Biol Med 1992; 199: 446-52.

32.- Bhaumik U, Aitken I, Kawachi I, Ringer S, Orav J, Lieberman E: Narrowing of sex differences in infant mortality in Massachusetts. J Perinatol 2004; 24: 949.

33.- Horbar JD, Badger GJ, Carpenter JH, Fanaroff AA, Kilpatrick $S$, Lacorte $M$, et al: Trends in mortality and morbidity for very low birth weight infants, 19911999. Pediatrics 2002; 110: 143-51.

34.- Crowley PA: Antenatal corticosteroid therapy: a metaanalysis of the randomized trials, 1972 to 1994. Am J Obstet Gynecol 1995; 173: 322-35.

35.- Yost CC, Soll RF: Early versus delayed selective surfactant treatment for neonatal respiratory distress syndrome. Cochrane Database Syst Rev 2000; CD001456.

36.- Ainsworth SB, Milligan DW: Surfactant therapy for respiratory distress syndrome in premature neonates: a comparative review. Am J Respir Med 2002; 1: 417-33.

37.- Soll RF, Blanco F: Natural surfactant extract versus synthetic surfactant for neonatal respiratory distress syndrome. Cochrane Database Syst Rev 2001; CD000144.

38.- Halliday HL: Natural vs synthetic surfactants in neonatal respiratory distress syndrome. Drugs 1996; 51: 226-37. 